\title{
In Memoriam: Ingo Wegener
}

\author{
Benjamin Doerr · Frank Neumann
}

Published online: 17 November 2009

(C) The Author(s) 2009. This article is published with open access at Springerlink.com

With deep sadness, we had to realize that our co-editor and co-founder of the theory track, Ingo Wegener, has died on the 26th of November 2008 after a long fight with cancer. His death is a tragic loss for all who knew him, friends, colleagues and students.

Ingo Wegener was born on the 4th of December 1950 in Bremen, Germany. He was a full professor at the Technische Universität Dortmund, leading a strong and influential group on efficient algorithms and complexity theory. He and his group contributed to many research areas. Their fundamental results on the complexity of Boolean functions and on the theory of evolutionary computation had an enormous impact. Ingo Wegener received numerous honors. Being appointed to the German Council of Science and Humanities as well as receiving the most important and prestigious German award for computer scientists, the Konrad-Zuse-Medaille, are just two examples.

In the field of evolutionary computation, Ingo Wegener established a completely new research direction. He started analyzing evolutionary algorithms by purely theoretical means. To substantiate a claim, we would look for a mathematical proof, valid for all inputs, rather than experimentally analyzing a limited number of examples. Unlike in the classical theory of algorithms community, this approach was uncommon of in the field of evolutionary computation.

With persistence and with convincing results he succeeded in getting his mathematical approach become a recognized direction in the field. Less than ten years

B. Doerr $(\bowtie) \cdot$ F. Neumann

Algorithms and Complexity Department, Campus E1 4, Max-Planck-Institut für Informatik, 66123 Saarbrücken, Germany

e-mail: doerr@mpi-inf.mpg.de

F. Neumann

e-mail: fne@mpi-inf.mpg.de 
after the publication of his first result of this kind, the new research area was mature enough to call for its own track at one of the leading conferences on evolutionary computation. Since 2007, the annual Genetic and Evolutionary Computation Conference (GECCO) hosts a track exclusively dealing with theory.

Even in the time when his illness took most of his energy, he followed the development of the track, read the papers submitted, and co-edited this special issue. It remains sad, though, that he was not able anymore to attend 'his' track.

We will deeply miss Ingo Wegener as a friend, scientist and colleague. 